\title{
Effects of periodic potentials on the critical velocity of superfluid Fermi gases in the BCS-BEC crossover
}

\author{
Gentaro Watanabe, ${ }^{1,2}$ Franco Dalfovo, ${ }^{3}$ Lev P. Pitaevskii, ${ }^{3,4}$ and Sandro Stringari ${ }^{3}$ \\ ${ }^{1}$ Asia Pacific Center for Theoretical Physics (APCTP), POSTECH, \\ San 31, Hyoja-dong, Nam-gu, Pohang, Gyeongbuk 790-784, Korea \\ ${ }^{2}$ Nishina Center, RIKEN, 2-1 Hirosawa, Wako, Saitama 351-0198, Japan \\ ${ }^{3}$ INO-CNR BEC Center and Department of Physics, University of Trento, 38123 Povo, Italy \\ ${ }^{4}$ Kapitza Institute for Physical Problems, 119334 Moscow, Russia
}

(Dated: June 20, 2018)

\begin{abstract}
We study the effects of an external periodic potential on the critical velocity of a superfluid Fermi gas in the crossover between the Bardeen-Cooper-Schrieffer (BCS) phase and Bose-Einstein condensation (BEC). We numerically solve the Bogoliubov-de Gennes equations to model a threedimensional (3D) gas of ultracold atoms in the superfluid phase flowing through a 1D optical lattice. We find that when the recoil energy is comparable to the Fermi energy, the presence of the periodic potential reduces the effect of pair-breaking excitations. This behavior is a consequence of the peculiar band structure of the quasiparticle energy spectrum in the lattice. When the lattice height is much larger than the Fermi energy, the periodic potential makes pairs of atoms to be strongly bound even in the BCS regime and pair-breaking excitations are further suppressed. We have also found that when the recoil energy is comparable to or larger than the Fermi energy, the critical velocity due to long-wavelength phonon excitations shows a non-monotonic behavior along the BCS-BEC crossover.
\end{abstract}

PACS numbers: 03.75.Ss, 03.75.Lm, 67.85.De

\section{INTRODUCTION}

Ultracold atom gases in optical lattices have been continuously attracting great interest for the last ten years [1 3]. Recent developments in the field of ultracold atom gases provide a new research arena in the physics of quantum fluids: by using Feshbach resonances of ultracold Fermi atoms, one can study the crossover from the Bardeen-Cooper-Schrieffer (BCS) phase to a BoseEinstein condensate (BEC) of molecules [4]. In the current research frontier, superfluidity of ultracold Fermi gases in optical lattices is very intriguing problem, which has interesting connections with similar issues in solid state physics, nuclear physics, and astrophysics.

The critical velocity of superflow due to energetic instability is one of the most important properties of superfluids, which has been pioneered by Landau [5]. If the velocity of superflow exceeds some critical value, the kinetic energy of the superfluid can be dissipated by creating excitations [5 8]. In uniform superfluid Fermi gases in the BCS-BEC crossover, excitations which cause the energetic instability are of two types: fermionic pair-breaking excitations in the BCS regime and longwavelength phonon excitations in the BEC regime [9, 10]. In the unitary regime both mechanisms are suppressed and the critical velocity shows a maximum value [9 11].

Recently, effects of periodic potentials on the critical velocity of Fermi superfluids has been studied experimentally [12. This experiment has stimulated theoretical investigations of this problem $13-16]$. Most of them has focused on the BCS regime in tight-binding approximation 13 15]. The purpose of the present work is to obtain an understanding of the critical velocity from a unified point of view covering all regions along the BCS-BEC crossover and both the strong and weak lattice regime. To this purpose, we use the Bogoliubov-de Gennes (BdG) equations. This theory accounts for both types of excitations which are relevant in this problem. In our previous work [16] we already used it for a gas at unitarity; here we extend the calculations in order to explore the whole crossover region. As a main result, we find that, when the lattice height is comparable to or much larger than the Fermi energy, the periodic potential reduces the effect of pair-breaking excitations. This is due to the periodic structure of the quasiparticle energy spectrum in the Brillouin zone and the formation of the bound molecules induced by the lattice. Another main result is that when the recoil energy is comparable to or larger than the Fermi energy, the critical velocity due to longwavelength phonon excitations shows a non-monotonic behavior along the BCS-BEC crossover. These effects are unique for Fermi superfluids in periodic potentials and do not exist in the case of single barrier potentials [16 18].

This paper is organized as follows. In Sec. II we explain the basic formalism employed in the present work. Then we show the results in Sec. III. Finally, summary and outlook are given in Sec. IV.

\section{BASIC FORMALISM}

We want to study the effect of the periodic potential on the Landau critical velocity of Fermi superfluids in the whole BCS-BEC crossover, in situations where the Fermi energy is larger or smaller than the lattice height. For 
this aim, we need to use a theoretical framework which can account for the formation of bound molecules induced by the periodic potential, which is important when the lattice height is larger than the Fermi energy [19, 20]; the same formalism must also account for pair-tunneling processes, which are important on the BEC side of the resonance [20, 21]. A suitable approach consists of the numerical solution of the Bogoliubov-de Gennes (BdG) equations [22]:

$$
\left(\begin{array}{cc}
H^{\prime}(\mathbf{r}) & \Delta(\mathbf{r}) \\
\Delta^{*}(\mathbf{r}) & -H^{\prime}(\mathbf{r})
\end{array}\right)\left(\begin{array}{c}
u_{i}(\mathbf{r}) \\
v_{i}(\mathbf{r})
\end{array}\right)=\epsilon_{i}\left(\begin{array}{c}
u_{i}(\mathbf{r}) \\
v_{i}(\mathbf{r})
\end{array}\right),
$$

where $u_{i}$ and $v_{i}$ are quasiparticle amplitudes and $\epsilon_{i}$ the corresponding eigen-energies. The single-particle hamiltonian is $H^{\prime}(\mathbf{r})=-\hbar^{2} \nabla^{2} / 2 m+V_{\text {ext }}-\mu$, where $m$ is the atom mass and $V_{\text {ext }}(\mathbf{r})$ is the external potential. The order parameter (or gap parameter) $\Delta(\mathbf{r})$ and the chemical potential $\mu$, appearing in Eq. (1), are variational parameters determined from the gap equation,

$$
\Delta(\mathbf{r})=-g \sum_{i} u_{i}(\mathbf{r}) v_{i}^{*}(\mathbf{r})
$$

together with the constraint

$$
n_{0}=\frac{2}{V} \sum_{i} \int\left|v_{i}(\mathbf{r})\right|^{2} d \mathbf{r}
$$

enforcing the conservation of the average density $n_{0}$. Here $g$ is the coupling constant for the contact interaction and $V$ is the volume of the system. The BdG eigenfunctions obey the normalization condition $\int d^{3} r\left[u_{i}^{*}(\mathbf{r}) u_{j}(\mathbf{r})+v_{i}^{*}(\mathbf{r}) v_{j}(\mathbf{r})\right]=\delta_{i, j}$. Finally, the energy density $e$ can be calculated as

$$
e=\frac{1}{V} \int d \mathbf{r} \sum_{i}\left[2\left(\mu-\epsilon_{i}\right)\left|v_{i}(\mathbf{r})\right|^{2}+\Delta^{*}(\mathbf{r}) u_{i}(\mathbf{r}) v_{i}^{*}(\mathbf{r})\right]
$$

In the present study, we consider a three-dimensional superfluid Fermi gas, which is uniform in the $x$ and $y$ directions and subject to a one-dimensional optical lattice along $z$ :

$$
V_{\mathrm{ext}}(z)=s E_{\mathrm{R}} \sin ^{2} q_{\mathrm{B}} z \equiv V_{0} \sin ^{2} q_{\mathrm{B}} z .
$$

Here $V_{0} \equiv s E_{\mathrm{R}}$ is the lattice height, $s$ is the laser intensity in dimensionless units, $E_{\mathrm{R}}=\hbar^{2} q_{\mathrm{B}}^{2} / 2 m$ is the recoil energy, $q_{\mathrm{B}}=\pi / d$ is the Bragg wave vector, and $d$ is the lattice constant. For practical reasons, throughout this paper, we set $s=1$ except for special cases, which we shall mention explicitly. The ratio between the Fermi energy and the lattice height is then varied by changing the average density of the gas.

In the presence of a supercurrent with wave vector $Q=$ $P / \hbar$ moving in the direction of the periodic potential, one can write the gap parameter in the form

$$
\Delta(\mathbf{r})=e^{i 2 Q z} \tilde{\Delta}(z)
$$

where $\tilde{\Delta}(z)$ is a complex function with period $d$. Therefore, from the gap equation, we see that the eigenfunctions of Eq. (1) must have the Bloch form $u_{i}(\mathbf{r})=$ $\tilde{u}_{i}(z) e^{i Q z} e^{i \mathbf{k} \cdot \mathbf{r}}$ and $v_{i}(\mathbf{r})=\tilde{v}_{i}(z) e^{-i Q z} e^{i \mathbf{k} \cdot \mathbf{r}}$. The wave vector $k_{z}$ lies in the first Brillouin zone (i.e., $-q_{\mathrm{B}} \leq k_{z} \leq q_{\mathrm{B}}$ ) and $\tilde{u}_{i}$ and $\tilde{v}_{i}$ are periodic in $z$ with period $d$. We also define the quasi-momentum $P_{\text {edge }}$ and quasi-wavenumber $Q_{\text {edge }}$ at the edge of the Brillouin zone for $P$ and $Q$ as $P_{\text {edge }}=\hbar Q_{\text {edge }} \equiv \hbar q_{\mathrm{B}} / 2$. (Note that the edge of the first Brillouin zone for $P$ is at $P_{\text {edge }}=q_{\mathrm{B}} / 2$ while that for $k_{z}$ is at $q_{\mathrm{B}}$.) This Bloch decomposition transforms Eq. (1) into the following $\mathrm{BdG}$ equations for $\tilde{u}_{i}$ and $\tilde{v}_{i}$ :

$$
\left(\begin{array}{cc}
\tilde{H}_{Q}^{\prime}(z) & \tilde{\Delta}(z) \\
\tilde{\Delta}^{*}(z) & -\tilde{H}_{-Q}^{\prime}(z)
\end{array}\right)\left(\begin{array}{c}
\tilde{u}_{i}(z) \\
\tilde{v}_{i}(z)
\end{array}\right)=\epsilon_{i}\left(\begin{array}{c}
\tilde{u}_{i}(z) \\
\tilde{v}_{i}(z)
\end{array}\right),
$$

where

$\tilde{H}_{Q}^{\prime}(z) \equiv \frac{\hbar^{2}}{2 m}\left[k_{x}^{2}+k_{y}^{2}+\left(-i \partial_{z}+Q+k_{z}\right)^{2}\right]+V_{\text {ext }}(z)-\mu$.

Here, the label $i$ represents the wave vector $\mathbf{k}$ as well as the band index. In order to remove the ultraviolet divergences in the BdG equations with contact potentials, we use the regularization scheme proposed by Refs. 23, 24]. Since we need to calculate the second derivatives of the energy with respect to the density and the quasi-momentum, we use large values of the cutoff energy $E_{C}$, especially in the BEC side, where the size of the pair is much smaller than the average inter-atomic distance (details are described in Sec. III).

As discussed in Refs. [9, 10], the energetic instability of superfluids of dilute Fermi gases can be caused by two processes [25]: the creation of long-wavelength superfluid phonon excitations or fermionic pair-breaking excitations. The critical velocity by the former process can be determined by the hydrodynamic analysis of the excitations 7, 16, 28 30. Starting from the continuity equation and the Euler equation, and linearizing with respect to the perturbations of the density and the velocity fields, we obtain the dispersion relation of the long-wavelength phonon,

$$
\omega(q)=\frac{\partial^{2} e}{\partial n_{0} \partial P} q+\sqrt{\frac{\partial^{2} e}{\partial n_{0}^{2}} \frac{\partial^{2} e}{\partial P^{2}}}|q| .
$$

Here, $\hbar \omega$ and $q$ are the energy and the wavenumber of the excitations, $n_{0}$ and $P$ are the average density and the quasimomentum of the superfluids. The energetic instability occurs when $\omega(q)$ becomes negative:

$$
\frac{\partial^{2} e}{\partial n_{0} \partial P}=\sqrt{\frac{\partial^{2} e}{\partial n_{0}^{2}} \frac{\partial^{2} e}{\partial P^{2}}}
$$

In practice, we calculate the energy density $e\left(n_{0}, P\right)$ for a given $n_{0}$ and $P$ from Eq. (44) using the solution of the BdG equations (7). Then the critical quasimomentum $P_{c}$ at which the energetic instability occurs is determined by 


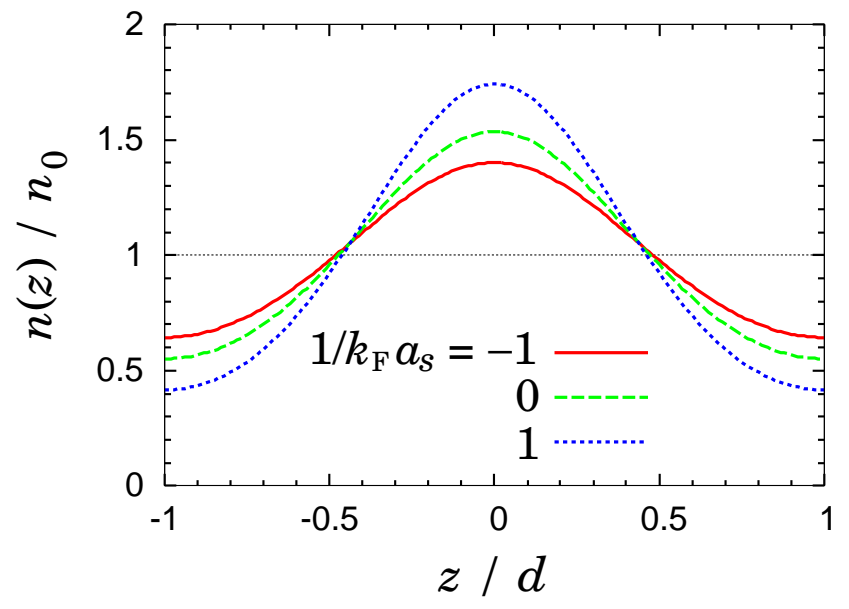

FIG. 1: (Color online) Density profile $n(z)$ at $P=0$ in the BCS-BEC crossover for $E_{\mathrm{F}} / E_{\mathrm{R}}=1$ and $s=1$. Here, $n(z)$ is normalized by the average density $n_{0}$

Eq. (10) [31]. We finally obtain the critical velocity $v_{c}$ from

$$
v_{c}=\frac{1}{n_{0}}\left(\frac{\partial e}{\partial P}\right)_{P_{c}} .
$$

On the other hand, the critical velocity due to the pair-breaking fermionic excitations can be determined by looking at the quasiparticle energy spectrum $\epsilon_{i}$. The energetic instability by the pair-breaking excitations occurs when some quasiparticle energy $\epsilon_{i}$ starts to be negative:

$$
\epsilon_{i} \leq 0
$$

From Eq. 111) evaluated at the critical quasi-momentum determined by this condition, we obtain a critical velocity for the pair-breaking excitations. The actual critical velocity of the system is the lowest between the ones obtained from the above two conditions 32]. We finally note that the gas becomes unstable also when some excitation energy starts to have a non-zero imaginary part. This corresponds to a dynamical instability, which causes an exponential growth of the amplitude of the perturbation. To address the problem of dynamical instability, short-wavelength bosonic excitations should be also properly included. This is beyond the scopes of the present work, in which we instead focus on the energetic instability. Results of the critical velocity for dynamical instability due to long-wavelength excitations are given in Appendix A.

\section{RESULTS}

We study the three cases of $E_{\mathrm{F}} / E_{\mathrm{R}}=2.5,1$, and 0.1 with a fixed value of $s=1$ except for a few cases which we shall mention explicitly. Here $E_{\mathrm{F}}=\hbar^{2} k_{\mathrm{F}}^{2} /(2 m)$ and $k_{\mathrm{F}}=\left(3 \pi^{2} n_{0}\right)^{1 / 3}$ are the Fermi energy and momentum, respectively, of a uniform noninteracting Fermi gas of density $n_{0}$. For each value of $E_{\mathrm{F}} / E_{\mathrm{R}}$, we solve the $\mathrm{BdG}$ equations for several values of the parameter $1 / k_{\mathrm{F}} a_{s}$ along the crossover from the BCS to the BEC side, namely $1 / k_{\mathrm{F}} a_{s}=-1,-0.5,0,0.5$, and 1 , where $a_{s}$ is the $s$-wave scattering length of atoms.

In the $x$ and $y$ directions, we assume periodic boundary conditions with period $L_{\perp}=12 \pi k_{\mathrm{F}}^{-1}$. We set the cutoff energy $E_{C}$ as follows: for $E_{\mathrm{F}} / E_{\mathrm{R}}=2.5, E_{C}=40 E_{\mathrm{F}}$ in the BCS side and $E_{C}=100 E_{\mathrm{F}}$ at unitarity and in the BEC side; for $E_{\mathrm{F}} / E_{\mathrm{R}}=1, E_{C}=50 E_{\mathrm{F}}$ in the BCS side and $E_{C}=100 E_{\mathrm{F}}$ at unitarity and in the BEC side; for $E_{\mathrm{F}} / E_{\mathrm{R}}=0.1, E_{C}=250 E_{\mathrm{F}}$ in the BCS side, $E_{C}=$ $350 E_{\mathrm{F}}$ at unitarity, and $E_{C}=500 E_{\mathrm{F}}$ in the BEC side.

\section{A. Density profiles and gap parameter}

In Fig. 11, we show the density profile $n(z)=$ $2 \sum_{i}\left|\tilde{v}_{i}(z)\right|^{2}$ of Fermi atoms at $P=0$ along the BCSBEC crossover for $E_{\mathrm{F}} / E_{\mathrm{R}}=1$. Moving from the BCS regime $\left(1 / k_{\mathrm{F}} a_{s}=-1\right)$ to the $\mathrm{BEC}$ regime $\left(1 / k_{\mathrm{F}} a_{s}=1\right)$, the density $n(z)$ becomes more inhomogeneous. This behavior is consistent with the fact that the compressibility of a uniform Fermi gas is known to increase monotonically with $1 / k_{\mathrm{F}} a_{s}$. We observe the same qualitative behavior for the other values of $E_{\mathrm{F}} / E_{\mathrm{R}}$.

In Fig. 2, we show the amplitude $|\Delta(z)|$ of the order parameter at $P=0$ for different values of $1 / k_{\mathrm{F}} a_{s}$ and $E_{\mathrm{F}} / E_{\mathrm{R}}$. From this figure one can see that, especially in the BCS regime $\left(1 / k_{\mathrm{F}} a_{s}=-1\right)$, the order parameter $|\Delta|$ is enhanced when the Fermi energy is smaller than the lattice strength, as shown by the blue curves for $E_{\mathrm{F}} / E_{\mathrm{R}}=0.1$ (i.e., $E_{\mathrm{F}} / V_{0}=0.1$ ). We understand this fact as due to the formation of bosonic molecules induced by the external periodic potential. This process is indeed expected to become significant when the lattice is strong [20].

\section{B. Critical velocity}

Before presenting the numerical results for the critical velocity, let us discuss the conditions upon which the periodic potential can produce significant effects on the behavior of long-wavelength phonons and pair-breaking excitations. For long-wavelength phonons the condition is $E_{\mathrm{F}} / E_{\mathrm{R}} \gtrsim 1$ or $s \gg 1$. In fact, in the opposite case, $E_{\mathrm{F}} / E_{\mathrm{R}} \ll 1$ and $s \lesssim 1$, the right-hand side of Eq. (10), which coincides with the sound speed at $P=0$, is much smaller than the maximum value of the left-hand side of Eq. (10), which is of order $q_{\mathrm{B}} / m$ (see also Fig. 5 in Ref. [16]). Thus Eq. (10] is satisfied at $P \simeq 0$ and the critical velocity is basically determined by the sound speed at $P=0$, which is also close to the sound speed in the uniform system. Consequently, the critical velocity due to long-wavelength phonons is almost unaf- 


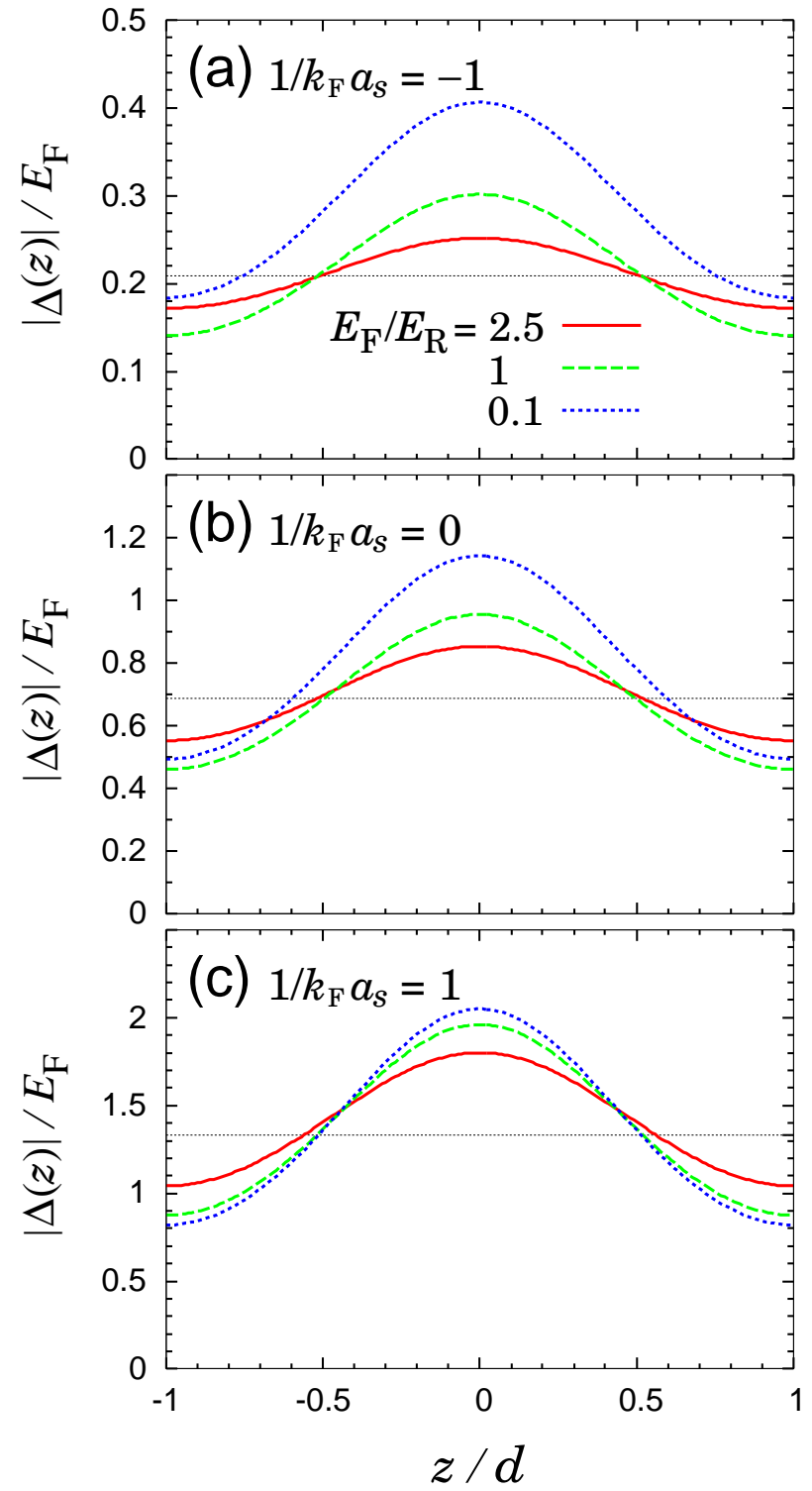

FIG. 2: (Color online) Amplitude $|\Delta(z)|$ of the gap parameter at $P=0$ in the BCS-BEC crossover: $1 / k_{\mathrm{F}} a_{s}=-1$ (a), 0 (b), and 1 (c). The horizontal dotted lines show the amplitude of the gap parameter for the uniform system at the same value of $1 / k_{\mathrm{F}} a_{s}$.

fected by the presence of the lattice if $E_{\mathrm{F}} / E_{\mathrm{R}} \ll 1$ and $s \lesssim 1$, even though the lattice height $V_{0}$ is large compared to the Fermi energy $E_{\mathrm{F}}$. On the other hand, the sufficient condition for pair-breaking excitations to be affected by the periodic potential due to the formation of bound molecules is $E_{\mathrm{F}} / V_{0} \ll 1$. This condition can be satisfied either by decreasing $E_{\mathrm{F}} / E_{\mathrm{R}}$ or by increasing $s$. When $E_{\mathrm{F}} / E_{\mathrm{R}} \simeq 1$, the critical velocity for pair-breaking excitations is also affected by a peculiar band structure of the quasi-particle spectrum.

Our results for the critical velocity $v_{c}$ are shown in Fig. 3 for $s=1$. Let us first concentrate on the results at high density, $E_{\mathrm{F}} / E_{\mathrm{R}}=2.5$, in panel (a). The open circles correspond to the critical velocity for long-wavelength phonons, which exhibits a non-monotonic behavior. In particular, in the BCS regime (negative $1 / k_{\mathrm{F}} a_{s}$ ) this critical velocity is strongly reduced compared to the one in a uniform gas (see, e.g., Fig. 8 in Ref. [10]). This is a peculiar effect of the lattice. However, for the parameters of Fig. 3(a), the actual critical velocity in the BCS regime is still given by fermionic pair-breaking excitations (filled squares). The latter are almost unaffected by the lattice and therefore, the overall behavior of the critical velocity in the crossover is qualitatively similar to that of a uniform gas, already discussed in Ref. [10]: in the BCS regime, $v_{c}$ increases when approaching unitarity $\left(1 / k_{\mathrm{F}} a_{s}=0\right)$, because the intra-pair attraction becomes stronger and thus the amplitude of the gap parameter increases; in the opposite BEC regime $\left(1 / k_{\mathrm{F}} a_{s}>0\right)$, the critical velocity is given by long-wavelength phonons and an increase of the inter-pair repulsion leads to a larger sound speed and, again, a critical velocity $v_{c}$ increases towards unitarity. As a consequence, $v_{c}$ takes a maximum value at $1 / k_{\mathrm{F}} a_{s} \simeq 0$.

When the recoil energy is comparable to the Fermi energy, the periodic potential causes qualitative changes in the results of the critical velocity. For $E_{\mathrm{F}} / E_{\mathrm{R}}=1$ [Fig. 33(b)], we observe that, at $1 / k_{\mathrm{F}} a_{s}=-0.5$, the critical velocity is given by long-wavelength phonon excitations rather than pair-breaking excitations even in the $\mathrm{BCS}$ regime. We understand this effect as mainly due to a peculiar band structure of the quasiparticle energy spectrum. In Fig. 4, we show the lowest band of the quasiparticle energy spectrum $\epsilon_{i}$ for the first radial branch with $k_{\perp}^{2} \equiv k_{x}^{2}+k_{y}^{2}=0$ at $P=0$ and $1 / k_{F} a=-0.5$. In general, the quasiparticle spectrum near the center of the Brillouin zone, at $\left|k_{z}\right| \simeq 0$, is only weakly affected by the periodicity of the system and hence the change of $\epsilon_{i}$ with increasing $P$ is close to that in the uniform system, given by the Doppler term $P \hbar k_{z} / m$. On the other hand, close to the zone edge, at $k_{z} \simeq \pm q_{\mathrm{B}}$, the change of $\epsilon_{i}$ with increasing $P$ is much smaller than $P \hbar k_{z} / m$ because of the periodicity of the Brillouin zone $\left(\epsilon_{i}\right.$ at $k_{z}= \pm q_{\mathrm{B}}$ must be identical). In the case of $E_{\mathrm{F}} / E_{\mathrm{R}}=1$, the minimum of $\epsilon_{i}$ is indeed located close to the edge of the Brillouin zone, unlike the other two cases of $E_{\mathrm{F}} / E_{\mathrm{R}}=2.5$ and 0.1 . Therefore, the reduction of the minimum value of $\epsilon_{i}$ with increasing $P$ is relatively small for $E_{\mathrm{F}} / E_{\mathrm{R}}=1$ and this is why we find that $v_{c}$ is determined by phononic instead of fermionic excitations in this case.

For smaller density $\left(E_{\mathrm{F}} / E_{\mathrm{R}}=0.1\right)$, we observe a significant increase of $v_{c}$ in the whole crossover [Fig. 3)(c)]. In our previous article [16], we already showed that, in a unitary Fermi superfluid with $E_{\mathrm{F}} / E_{\mathrm{R}} \ll 1$, the phononic critical velocity is almost unaffected by the lattice, if $s \lesssim 1$, and remains close to the speed of sound of a uniform gas with the same density 34. This can be understood by recalling that phonons always have wavelength larger than the healing length of the superfluid, which is the order of $k_{\mathrm{F}}^{-1}$ or greater. When $E_{\mathrm{F}} / E_{\mathrm{R}} \ll 1$, 

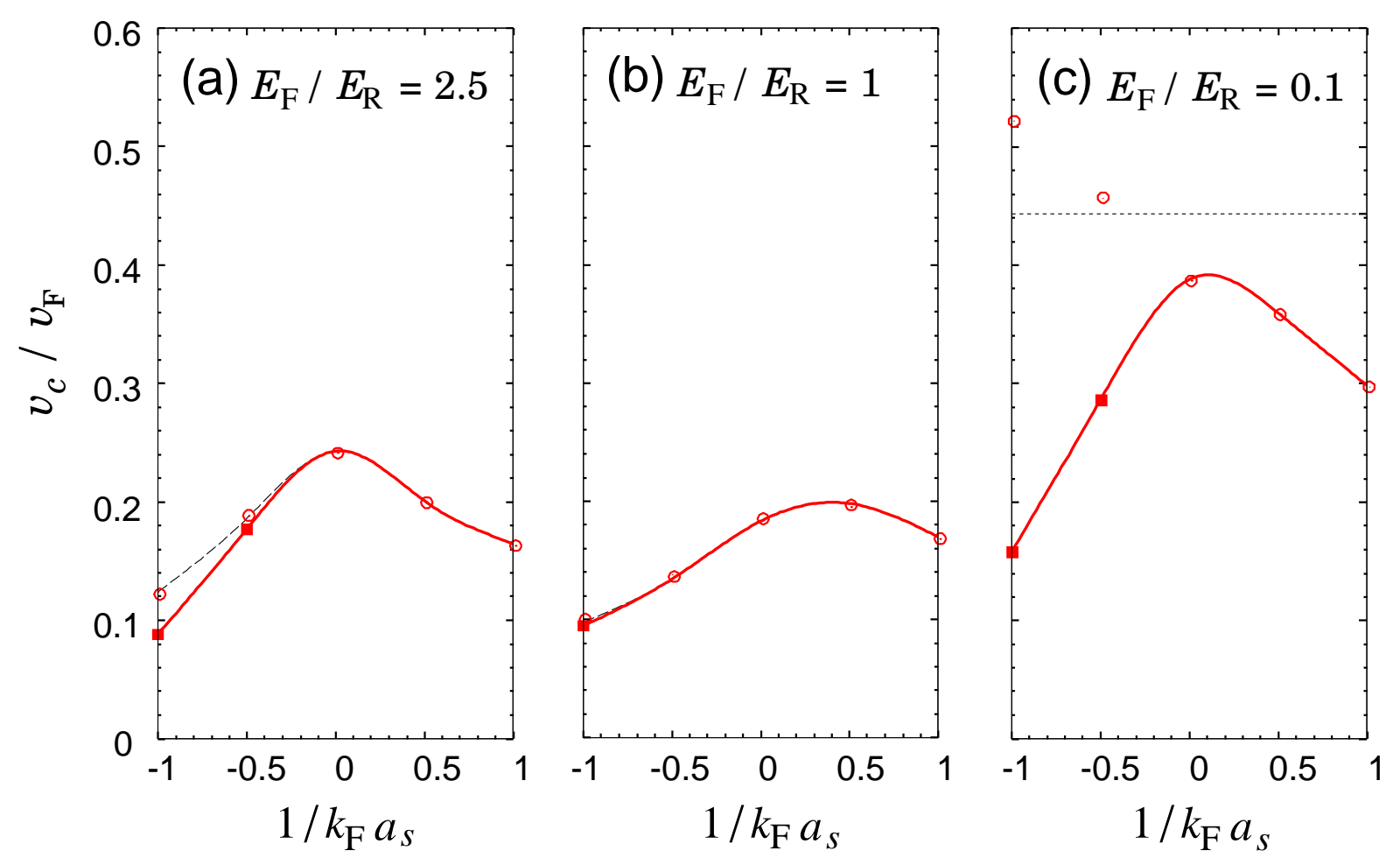

FIG. 3: (Color online) Critical velocity $v_{c}$ of the energetic instability for $E_{\mathrm{F}} / E_{\mathrm{R}}=2.5$ (a), 1 (b), and 0.1 (c) with $s=1$ in the BCS-BEC crossover. Open circles and filled squares show the critical velocity due to long-wavelength phonons and fermionic pair-breaking excitations, respectively. The horizontal dotted line in panel (c) represents the value of the sound velocity $c_{s}^{(0)}$ of uniform system at unitarity, $c_{s}^{(0)} / v_{\mathrm{F}}=(1+\beta)^{1 / 2} / \sqrt{3} \simeq 0.443$. The red solid lines and the black dashed lines are guides to the eye.

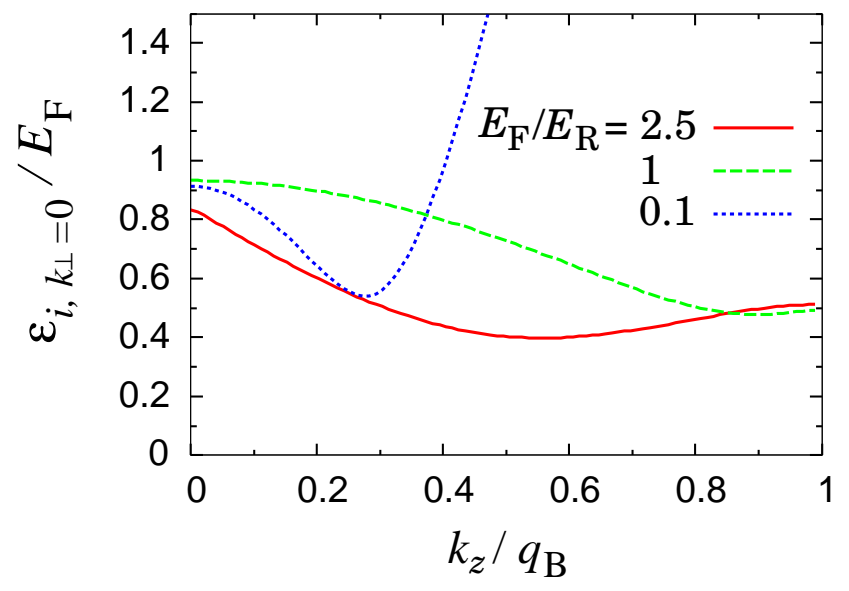

FIG. 4: (Color online) Lowest band of the quasiparticle energy spectrum $\epsilon_{i}$ for $P=0$ and $1 / k_{F} a=-0.5$. Here, we show the first radial branch with $k_{\perp}^{2} \equiv k_{x}^{2}+k_{y}^{2}=0$. For $E_{\mathrm{F}} / E_{\mathrm{R}}=1$, the minimum of $\epsilon_{i}$ is located close to the Brillouin zone edge $k_{z}=q_{\mathrm{B}}$.

the healing length becomes much larger than the lattice spacing $d=\pi / q_{\mathrm{B}}$, and this makes the phonons insensitive

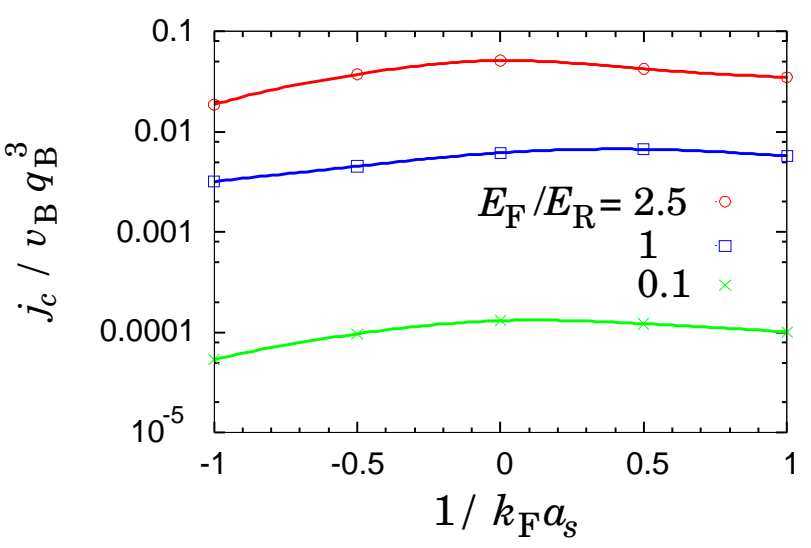

FIG. 5: (Color online) Critical current $j_{c}=n_{0} v_{c}$ for the same cases in Fig. 3. Here we show the lowest value of $j_{c}$, given either by long-wavelength phonon excitations or by fermionic pair-breaking excitations. The curves connecting symbols are guides to the eye.

to the lattice itself. In the present work, we find that the same is true even away from unitarity, leading to a larger critical velocity in the whole crossover [empty circles in 


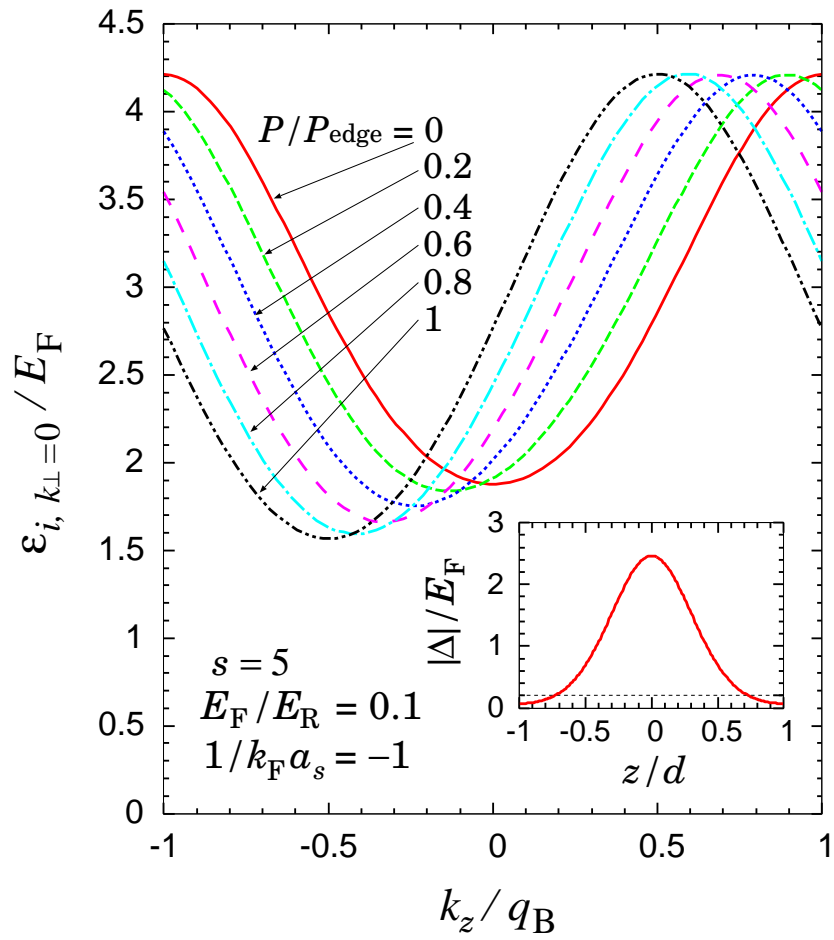

FIG. 6: (Color online) Lowest band of the quasiparticle energy spectrum $\epsilon_{i}$ for large lattice height with $s=5$ and $E_{\mathrm{F}} / E_{\mathrm{R}}=0.1$ (i.e., $E_{\mathrm{F}} / V_{0}=0.02$ ) in the BCS regime at $1 / k_{\mathrm{F}} a_{s}=-1$. Here, we show the first radial branch with $k_{\perp}^{2} \equiv k_{x}^{2}+k_{y}^{2}=0$, which always gives the smallest values of $\epsilon_{i}$ in this case. The inset shows the amplitude $|\Delta(z)|$ of the order parameter at $P=0$. The horizontal dotted line shows the amplitude of the order parameter for the uniform system at the same values of $1 / k_{\mathrm{F}} a_{s}=-1$.

Fig. [3(c)]. The critical velocity due to pair-breaking excitations (filled squares) is also increased because a lattice strength $V_{0}$ much larger than the Fermi energy gives a stronger attraction between paired atoms.

In Fig. 5 we show the critical current $j_{c}=n_{0} v_{c}$ for the same cases of Fig. 3. Due to the low density at $E_{\mathrm{F}} / E_{\mathrm{R}}=$ 0.1 , the critical current is much smaller than the other cases even though $v_{c}$ in units of $v_{\mathrm{B}}=q_{\mathrm{B}} / \mathrm{m}$ for $E_{\mathrm{F}} / E_{\mathrm{R}}=$ 0.1 is comparable to that of $E_{\mathrm{F}} / E_{\mathrm{R}}=1$.

\section{Dependence on the lattice height}

All results shown in Figs. 1,5 have been obtained by fixing the lattice height $s=1$ and vary the density in order to change the key parameter $E_{\mathrm{F}} / E_{\mathrm{R}}$. If we increase $s$ keeping the average density fixed, the superfluid flow is suppressed and $v_{c}$ is also reduced in general.

A systematic analysis as a function of $s$, which would be natural from the experimental viewpoint, is computationally very demanding and is beyond the scopes of this work. The choice of $s=1$ is not accidental, however. It turns out, in fact, that around $s=1$ the effects of the lattice on the critical velocity are the most pronounced as far as the interplay between pair-breaking and longwavelength bosonic excitations is concerned. For lower values of $s$ these two types of excitations behave qualitatively the same as in a uniform superfluid, as function of $1 / k_{F} a$, being scarcely affected by the lattice, at least within the range of $E_{\mathrm{F}} / E_{\mathrm{R}}$ considered in this work. On the other hand, at larger $s$ the pair-breaking instability is quickly suppressed and long-wavelength excitations becomes dominant along the crossover.

The reason why a strong lattice prevents the pairbreaking processes can be understood by looking at Fig. 6, where we show the quasiparticle energy spectra at various values of $P$ in the case of $s=5, E_{\mathrm{F}} / E_{\mathrm{R}}=0.1$ (i.e., $E_{\mathrm{F}} / V_{0}=0.02$ ), and $1 / k_{\mathrm{F}} a_{s}=-1$. The spectrum for $P=0$ shows a quadratic dependence of $k_{z}$ with a positive curvature around $k_{z}=0$ and there are no minima at $k_{z} \neq 0$. Even though the figure represents a case in the deep BCS regime, the structure of $\epsilon_{i}$ is consistent with the formation of bound pairs. In the inset of the same figure, we show the amplitude $|\Delta(z)|$ of the gap parameter at $P=0$. First, we note that the minimum value of $|\Delta(z)|$ at $z / d= \pm 1$ is smaller than, but still comparable to the value of $|\Delta|$ in the uniform case, suggesting that the system is indeed in the superfluid phase. More importantly, one sees a large enhancement of $|\Delta(z)|$, near $z=0$, compared to the uniform system, which shows the formation of bosonic bound molecules. A consequence of this lattice induced molecular formation is that the energetic instability due to pair-breaking excitations is suppressed and does not occur at any values of $P$.

For the same parameters of Fig. 6, the energetic instability due to long-wavelength phonons instead occurs at $P=0.226 \hbar q_{\mathrm{B}}=0.452 P_{\text {edge }}$ and the corresponding critical velocity at $1 / k_{\mathrm{F}} a_{s}=-1$ is $v_{c}=0.0662 v_{\mathrm{F}}$. We also find $v_{c}=0.0429 v_{\mathrm{F}}$ at unitarity and $v_{c}=0.0302 v_{\mathrm{F}}$ at $1 / k_{\mathrm{F}} a_{s}=1$. This means that, in the whole crossover, the critical velocity at $s=5$ is largely reduced compared to the red line in Fig. 3 (c) for $s=1$ and does not exhibit a maximum anymore. The behavior of $v_{c}$ as a function of $s$ at unitarity has been already discussed in Ref. [16].

\section{SUMMARY AND OUTLOOK}

We have studied the effects of a periodic potential on the Landau critical velocity of a Fermi superfluid in the BCS-BEC crossover. We have considered a 3D superfluid Fermi gas flowing in a 1D periodic potential produced by an optical lattice. Using the Bogoliubov-de Gennes equations, we have obtained a unifying picture both for weak and strong lattices and in the whole BCS-BEC crossover. We have found that, when the recoil energy is comparable to the Fermi energy, energetic instability due to fermionic pair-breaking excitations can be less effective as a consequence of the periodic structure of the quasiparticle energy spectrum. When the lattice height is much larger 
than the Fermi energy, pair-breaking excitations are prevented because the lattice potential gives a stronger attraction between paired atoms, eventually forming bound bosonic molecules. We have also found that, when the recoil energy is comparable to or larger than the Fermi energy, the critical velocity due to the long wavelength phonon excitations is drastically reduced by the lattice in the BCS regime leading to its non-monotonic behavior along the BCS-BEC crossover.

A further interesting issue regards the possible existence of roton-like minima in the bosonic dispersion curve. This excitations are obtained at low filling fractions and within a tight-binding attractive Hubbard model [14, 15, 26, 27]. The roton-like minima arise from strong charge-density-wave fluctuations. These fluctuations are expected to be less favored in our system, where the gas is uniform in the transverse directions. However, if such roton-like excitations exist also in the our case (3D gas in a 1D lattice), they would lower the critical velocity in the BCS regime and for strong lattices. To address this issue, one should use, for instance, a quasiparticle random phase approximation (QRPA) on top of the stationary solution of the Bogoliubov-de Gennes equations. This is an interesting challenge for future investigations.

Finally, we would like to discuss a similarity between the present system and nuclear "pasta" phases [36 38 in crusts of neutron stars. The pasta nuclei are those of exotic shapes such as rod-like and slab-like structures. In neutron star crusts, the pasta nuclei are immersed in background electrons and a gas of dripped neutrons, which is regarded to be in the superfluid phase. The setup considered in the present work resembles superfluid neutrons in the pasta phase with slab-like nuclei, which are in the normal phase and provide a $1 \mathrm{D}$ periodic potential for superfluid neutrons [39].

\section{Acknowledgments}

We thank Ki-Seok Kim, Chris Pethick, Robin G. Scott, and Tetsuya Takimoto for helpful discussions and comments. Calculations were performed on the RIKEN Integrated Cluster of Clusters (RICC) system, WIGLAF at the University of Trento, and BEN at ECT*. GW acknowledges the Max Planck Society (MPG), the Korea
Ministry of Education, Science and Technology (MEST), Gyeongsangbuk-Do, and Pohang City for the support of the Independent Junior Research Group at the Asia Pacific Center for Theoretical Physics (APCTP). This work, as a part of the European Science Foundation EUROCORES Program EuroQUAM-FerMix, is supported by funds from the CNR and the EC Sixth Framework Programme. It is also supported by MiUR.

\section{Appendix A: Dynamical instability within hydrodynamic analysis}

In this Appendix, we show the results of the critical velocity $v_{c}$ for dynamical instability due to longwavelength excitations. This instability occurs when a non-zero imaginary part appears in the excitation energy (9). The condition is

$$
\frac{\partial^{2} e}{\partial n_{0}^{2}} \frac{\partial^{2} e}{\partial P^{2}}<0
$$

Even though the long-wavelength excitations in general do not give the lowest critical velocity of the dynamical instability, this condition becomes useful at $V_{0} \gg E_{\mathrm{F}}$, when the $P$-dependence of $e$ is almost sinusoidal and the critical velocity dependents rather weakly on the wavelength of the excitations. In this case, the above condition gives an onset of the dynamical instability at $P=P_{\text {edge }} / 2$, which coincides with the condition $e(P+\hbar q)-e(P)=e(P)-e(P-\hbar q)$ for $\left.V_{0} \gg|\Delta| \underline{33}\right]$. This corresponds to the energy and momentum conservation for two particles decaying into two different Bloch states with $e(P \pm \hbar q)$.

In Fig. 7, we show the critical velocity for the dynamical instability determined by the condition (A1). The most striking feature is the large values of $v_{c}$ in the case of $s=1$ and $E_{\mathrm{F}} / E_{\mathrm{R}}=0.1$. As in the case of the energetic instability, this is due to the fact that phonons are insensitive to the lattice when $s \lesssim 1$ and $E_{\mathrm{F}} / E_{\mathrm{R}} \ll 1$, i.e., the healing length is much larger than the lattice spacing. Note that, even for the same value of $E_{\mathrm{F}} / E_{\mathrm{R}}=0.1$, the critical velocity can be rather small provided $s \gg 1$. This tendency is confirmed by the results for $s=5$ and $E_{F} / E_{R}=0.1$ shown by crosses in Fig. 7
[1] O. Morsch and M. Oberthaler, Rev. Mod. Phys. 78, 179 (2006).

[2] M. Lewenstein, A. Sanpera, V. Ahufinger, B. Damski, A. Sen, and U. Sen, Adv. Phys. 56, 243 (2007).

[3] I. Bloch, J. Dalibard, and W. Zwerger, Rev. Mod. Phys. 80, 885 (2008).

[4] S. Giorgini, L. P. Pitaevskii, and S. Stringari, Rev. Mod. Phys. 80, 1215 (2008).

[5] L. D. Landau, J. Phys. USSR 5, 71 (1941).

6] P. Nozières and D. Pines, The Theory of Quantum Liq- uids (Perseus, Cambridge, 1999), Vol. II.

[7] C. J. Pethick and H. Smith, Bose-Einstein Condensation in Dilute Gases 2nd ed. (Cambridge Univ. Press, New York, 2008).

[8] L. P. Pitaevskii and S. Stringari, Bose-Einstein Condensation (Clarendon, Oxford, 2003).

[9] N. Andrenacci, P. Pieri, and G. C. Strinati, Phys. Rev. B 68, 144507 (2003).

[10] R. Combescot, M. Yu. Kagan, and S. Stringari, Phys. Rev. A 74, 042717 (2006). 


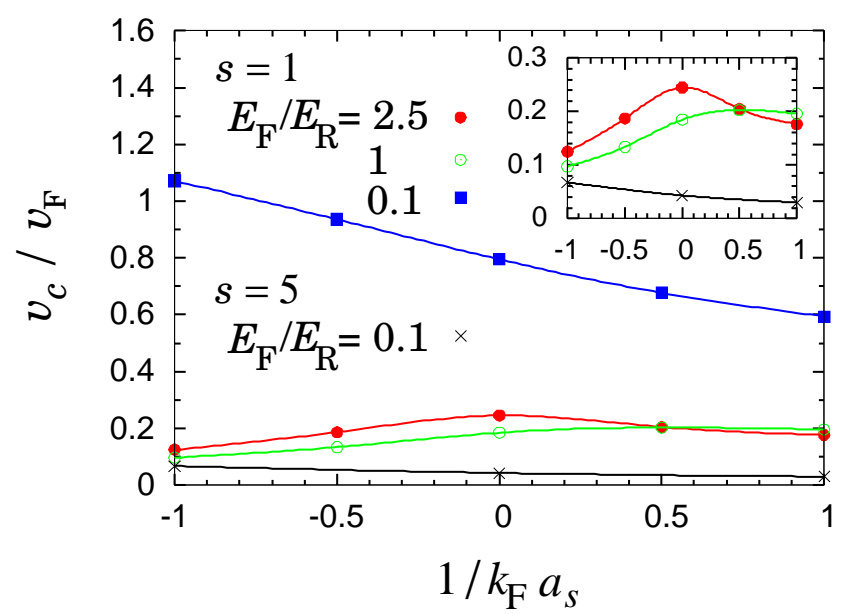

FIG. 7: (Color online) Critical velocity $v_{c}$ for dynamical instability due to long-wavelength excitations in the BCS-BEC crossover. The solid lines are guides to the eye. The inset shows the magnification of the region of $v_{c} / v_{\mathrm{F}}=0-0.3$.

[11] R. Sensarma, M. Randeria, and T.-L. Ho, Phys. Rev. Lett. 96, 090403 (2006).

[12] D. E. Miller, J. K. Chin, C. A. Stan, Y. Liu, W. Setiawan, C. Sanner, and W. Ketterle, Phys. Rev. Lett. 99, 070402 (2007).

[13] A. A. Burkov and A. Paramekanti, Phys. Rev. Lett. 100, 255301 (2008)

[14] Y. Yunomae, D. Yamamoto, I. Danshita, N. Yokoshi, and S. Tsuchiya, Phys. Rev. A 80, 063627 (2009).

[15] R. Ganesh, A. Paramekanti, and A. A. Burkov, Phys. Rev. A 80, 043612 (2009).

[16] G. Watanabe, F. Dalfovo, F. Piazza, L. P. Pitaevskii, and S. Stringari, Phys. Rev. A 80, 053602 (2009).

[17] A. Spuntarelli, P. Pieri, and G. C. Strinati, Phys. Rev. Lett. 99, 040401 (2007).

[18] A. Spuntarelli, P. Pieri, and G. C. Strinati, Phys. Rep. 488, 111 (2010).

[19] G. Orso, L. P. Pitaevskii, S. Stringari, and M. Wouters, Phys. Rev. Lett. 95, 060402 (2005).

[20] G. Watanabe, G. Orso, F. Dalfovo, L. P. Pitaevskii, and S. Stringari, Phys. Rev. A 78, 063619 (2008).

[21] Y. Ohashi, Phys. Rev. A 78, 063617 (2008).

[22] P. G. de Gennes, Superconductivity of Metals and Alloys (Benjamin, New York, 1966), Chap. 5, pp. 137-170.

[23] G. Bruun, Y. Castin, R. Dum, and K. Burnett, Eur. Phys. J. D 7, 433 (1999)

[24] A. Bulgac and Y. Yu, Phys. Rev. Lett. 88, 042504 (2002).

[25] Here, we ignore the possible existence of roton-like minima in the collective excitation spectrum obtained in the BCS regime for $1 \mathrm{D}(2 \mathrm{D}$ or $3 \mathrm{D})$ optical lattice in a $1 \mathrm{D}(2 \mathrm{D}$ or 3D) system using a tight-binding attractive Hubbard model [14, 15, 26, 27]. See also Sec. IV]

[26] J. O. Sofo, C. A. Balseiro, and H. E. Castillo, Phys. Rev. B 45, 9860 (1992)

[27] T. Kostyrko and R. Micnas, Phys. Rev. B 46, 11025 (1992).

[28] M. Machholm, C. J. Pethick, and H. Smith, Phys. Rev.
A 67, 053613 (2003).

[29] E. Taylor and E. Zaremba, Phys. Rev. A 68, 053611 (2003).

[30] L. P. Pitaevskii, S. Stringari, and G. Orso, Phys. Rev. A 71, 053602 (2005).

[31] Note that the partial derivatives in Eq. (10) should be done by keeping $a_{s}$ and $d$ constant.

[32] We notice that the meaning of Landau stability is different for the bosonic and fermionic branches of the spectrum. A violation of the Landau stability condition for bosonic excitations makes thermal equilibrium impossible when $v>v_{c}$, due to boundless creation of the excitations. Conversely, in the case of fermionic excitations, the flow cannot create more than one excitation for a given state due to Pauli principle. Thus a velocity greater than the critical one results in the appearance of a normal part of the fluid even at $T=0$. How this normal part manifests itself depends on the concrete experimental realization. For example, for a finite body moving in the fluid, the fermionic excitations produced via Landau instability would move away from the body, causing a friction. The investigation of this problem, however, is beyond the scope of the present work.

[33] B. Wu and Q. Niu, Phys. Rev. A 64, 061603(R) (2001).

[34] Note that the sound velocity at $P=0$ basically decreases with increasing $1 / k_{\mathrm{F}} a_{s}$ for all values of $E_{\mathrm{F}} / E_{\mathrm{R}}$. However, the velocity at which the energetic instability occurs due to the long-wavelength phonon excitations does not show a monotonic behavior with increasing $1 / k_{\mathrm{F}} a_{s}$ for $E_{\mathrm{F}} / E_{\mathrm{R}}=2.5$ and 1 , unlike $E_{\mathrm{F}} / E_{\mathrm{R}}=0.1$.

[35] Here we use $\beta=-0.41$ predicted by the mean-field BdG theory. We notice that $a b$-initio Monte Carlo simulations give instead $\beta=-0.58 \pm 0.01$. See J. Carlson, S.-Y. Chang, V. R. Pandharipande, and K. E. Schmidt, Phys. Rev. Lett. 91, 050401 (2003); G. E. Astrakharchik, J. Boronat, J. Casulleras, and S. Giorgini, Phys. Rev. Lett. 93, 200404 (2004); J. Carlson and S. Reddy, Phys. Rev. Lett. 95, 060401 (2005).

[36] D. G. Ravenhall, C. J. Pethick and J. R. Wilson, Phys. Rev. Lett. 50, 2066 (1983).

[37] M. Hashimoto, H. Seki, and M. Yamada, Prog. Theor. Phys. 71, 320 (1984).

[38] For recent works, see, e.g., G. Watanabe, H. Sonoda, T. Maruyama, K. Sato, K. Yasuoka, and T. Ebisuzaki, Phys. Rev. Lett. 103, 121101 (2009); H. Sonoda, G. Watanabe, K. Sato, K. Yasuoka, and T. Ebisuzaki, Phys. Rev. C 77, 035806 (2008), and references therein.

[39] In the case of the nuclear pasta phase in neutron stars, an estimate of the critical velocity can be obtained as follows. For the pasta phase with slab-like nuclei, the average neutron density $n_{0} \simeq 0.08 \mathrm{fm}^{-3}$ and the lattice constant $d \simeq 15-20 \mathrm{fm}$ and thus $E_{\mathrm{F}} / E_{\mathrm{R}}=\left(k_{\mathrm{F}} / q_{\mathrm{B}}\right)^{2} \sim 40-$ 70. The strength $V_{0}$ of the optical lattice corresponds to the depth of the bottom of the conduction band of neutrons measured from the average potential energy outside nuclei 40$]$. Since the neutron Fermi energy $E_{\mathrm{F}}$, in for the density of neutrons inside nuclei is $E_{\mathrm{F} \text {, in }} \sim 35-40 \mathrm{MeV}$ and the neutron chemical potential $\mu_{\text {in }}$ inside nuclei is $\mu_{\text {in }} \sim 10-15 \mathrm{MeV}$, we estimate $V_{0} \sim\left|\mu_{\text {in }}-E_{\mathrm{F}, \text { in }}\right| \sim 25$ $\mathrm{MeV}$. Thus, we obtain $s \sim 25-50$. Since the absolute value of the neutron scattering length $a_{s} \simeq-18.5 \mathrm{fm}$ is much larger than the radius $r_{s}$ of the average volume per neutron, $r_{s} \simeq 1.5 \mathrm{fm}$, superfluid neutrons in neutron star crusts are roughly approximated as a uni- 
tary Fermi gas [41, 43, 44] (see also Refs. 40, 45, 46]) Here, because $E_{\mathrm{F}} / E_{\mathrm{R}} \gg 1$, we can safely employ the hydrodynamic theory with the local density approximation given in Ref. 16 to estimate the critical velocity. Using Eq. (3) of Ref. 16] together with $\beta=-0.58$ [35] and the above parameters, we estimate $v_{c} \sim 10^{-3}-10^{-2} v_{\mathrm{F}}$ with $v_{\mathrm{F}} \simeq 0.3 c$, where $c$ is the speed of light.

[40] C. J. Pethick, private communication.

[41] Here, just to give an order of estimate, we shall ignore a quantitatively significant correction by the effective range $r_{e} \simeq 2.7 \mathrm{fm}$ [42], which is comparable to the interparticle separation between neutrons.

[42] A. Schwenk and C. J. Pethick, Phys. Rev. Lett. 95, 160401 (2005).

[43] G. F. Bertsch, in the announcement of the Tenth International Conference on Recent Progress in Many-Body Theories, (1999) unpublished.

[44] G. A. Baker, Jr., Phys. Rev. C 60, 054311 (1999).

[45] A. Gezerlis and J. Carlson, Phys. Rev. C 77, 032801(R) (2008).

[46] T. Abe and R. Seki, Phys. Rev. C 79, 054003 (2009). 